\title{
Generation of Ultrahigh-Speed Tunable-Rate Optical Pulses Using Strongly Gain-Coupled Dual-Wavelength DFB Laser Diodes
}

\author{
R. Hui, B. Zhu, K. Demarest, C. Allen, and Jin Hong
}

\begin{abstract}
A novel and simple method to generate a variablerate, ultrahigh-speed optical pulse train is demonstrated using a dual-wavelength, strongly gain-coupled distributed-feedback laser diode. The repetition rate of the optical pulse train is continuously tunable from 25 to $80 \mathrm{GHz}$ and no high-speed electronics are required in this method.
\end{abstract}

Index Terms-Nonlinear fiber optics, optical communications, optical solitons, semiconductor lasers.

G ENERATING high-repetition-rate optical pulse trains is required for the development of future ultrahighspeed optical transmission systems and for ultrahigh-speed all-optical signal processing [1]. Presently, complicated modelocking techniques are available for high-speed optical pulse generation using diode lasers [2], where pulses are generated at a fixed repetition rate, determined by the roundtrip time of the diode laser resonator. Another method is gain switching of laser diodes, which suffers from high time-jitter. Also pulse generation at $>50-\mathrm{GHz}$ repetition-rates is extremely difficult with this method because of the limitation of the device modulation bandwidth and RF supply [3].

High-repetition-rate optical pulses can also be generated using a dual-wavelength light source [4]. The beating between the two wavelengths generates a high-frequency sinusoidal signal. This sinusoidal signal can be compressed into optical pulses by passing it through a nonlinear fiber system. A common approach is to use two discrete laser diodes, which requires complicated synchronization schemes between these two lasers. Dual-wavelength operation can also be accomplished by selecting the appropriate phase modulation sidebands from an externally phase modulated light source [5]. This requires high-frequency modulation and optical filters. In order to make this method more practical, a simple photonic device that can simultaneously generate two stable wavelengths is desired. In this letter, we report the use of a multisection, strongly gain-coupled (SGC) distributedfeedback (DFB) laser diode as a dual-wavelength light source

Manuscript received December 14, 1998; revised January 26, 1999. This work was supported in part by Nortel, in part by Sprint, and in part by the National Science Foundation under Grant EPSCoR/ECS 963-2617.

R. Hui, B. Zhu, K. Demarest, and C. Allen are with the Information and Telecommunication Technology Center, Department of Electrical Engineering and Computer Science, The University of Kansas, Lawrence, KS 66045 USA.

J. Hong is with Advanced Technology, Nortel, Ottawa, ON, K1Y 4H7 Canada.

Publisher Item Identifier S 1041-1135(99)03600-9.

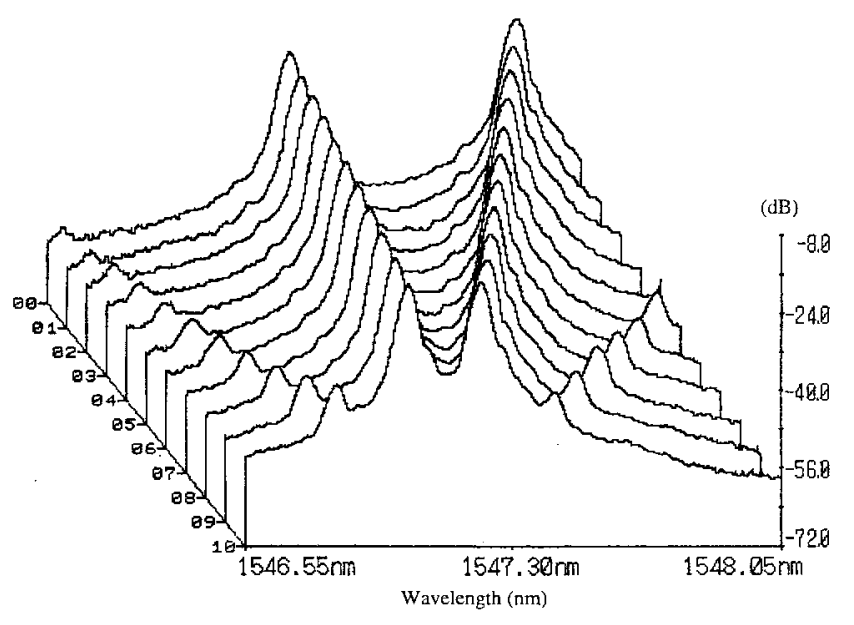

Fig. 1. Tuning characteristic of wavelength separation measured from a dual-wavelength laser diode.

to generate tunable ultrahigh-speed optical pulses. The repetition rate of the optical pulse trains is continuously tunable from 25 to $80 \mathrm{GHz}$ and beyond. No high-speed electronics is required in this high-speed optical pulse generator.

In order to realize the stable dual-wavelength operation in a single laser diode with multiple sections, a very strong gain-coupling was chosen in a DFB structure to minimize the effect of interaction between different laser sections [6]-[7]. In this way, two wavelengths, each originating from different laser sections, are mutually independent and the wavelength separation can be easily varied over a wide range by adjusting the injection currents. Since different laser sections are on the same chip, the wavelength difference is stable. The orientation of polarization is aligned, and both are insensitive to ambient temperature and mechanical variations. In this kind of laser diode, the wavelength separation between the two wavelengths can be designed to cover a large frequency range. The wavelength separation can also be fine-tuned by current injection.

The detailed structure of this laser is reported in [6], where six of the eight quantum wells in the active region were etched periodically to form a strong in-phase gaincoupling. Fig. 1 shows a typical current-tuning characteristic of the optical spectrum measured from such a dual-wavelength laser diode, when the wavelength separation is varied from 25 to $80 \mathrm{GHz}$. The two small peaks at each side of the main modes are four-wave-mixing (FWM) sidebands. They 


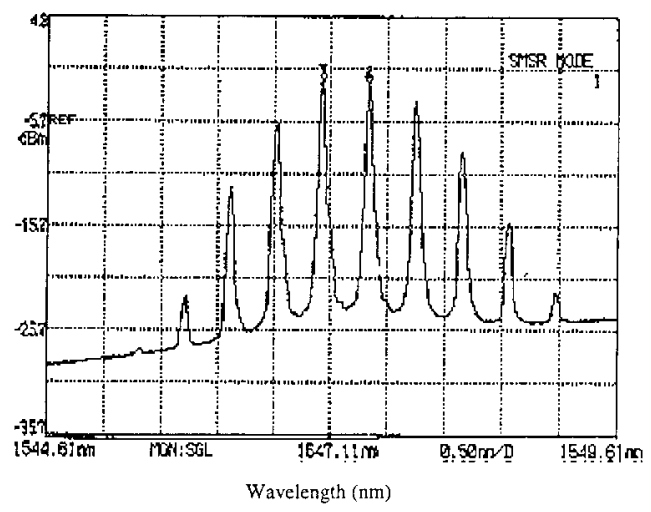

(a)

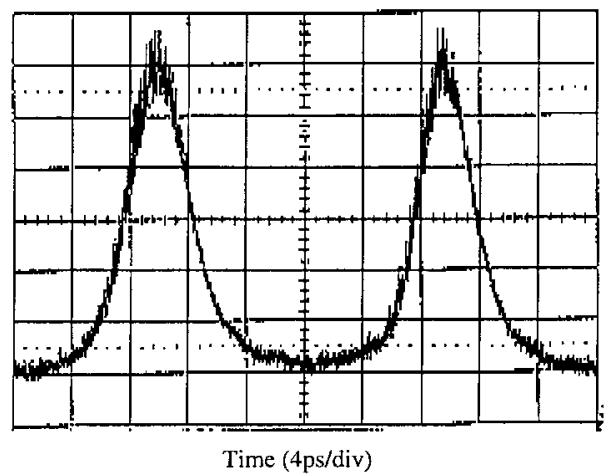

(b)

Fig. 2. (a) Optical spectrum measured after the dual-wavelength light travels through the two cascaded fiber sections. (b) The corresponding time domain waveform. Measured by an autocorrelator based on SHG. Horizontal scale: $4 \mathrm{ps} / \mathrm{div}$.

are created by population pulsation at the beat frequency of the two stable major modes inside the front laser section [8]. Since the wavelengths of these newly generated FWM components are at multiples of the main-mode wavelength separation, they can be regarded as higher order harmonics of the beating signal between the two main-modes. In practice, there is no limitation for the maximum wavelength separation. However, the minimum wavelength separation is limited by the effect of optical injection-locking [9]. In order to ensure stable dual-wavelength operation and keep away from mutual injection-locking between the two wavelengths, the lower limitation of wavelength separation is typically of the order of $20 \mathrm{GHz}$.

It is worth noting that this two-mode operation is fundamentally different from the mode-partitioning found in conventional multimode laser diodes, where modes are competing between each other. In order to verify that there was no competition between the two wavelengths in our laser, a tunable optical filter was used to select only one of the two wavelengths. The output optical power from the optical filter was measured by a high-speed photodiode, amplified, and monitored by a $1-\mathrm{GHz}$ oscilloscope. No significant power fluctuation was observed. Therefore, we concluded that mode competition does not exist in this dual-wavelength laser diode.

In order to verify that this beating sinusoidal signal can be compressed into short optical pulses using fiber nonlinearity

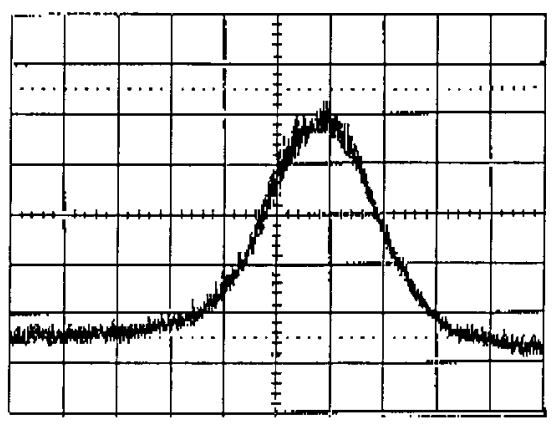

Time (4ps/div)

(a)

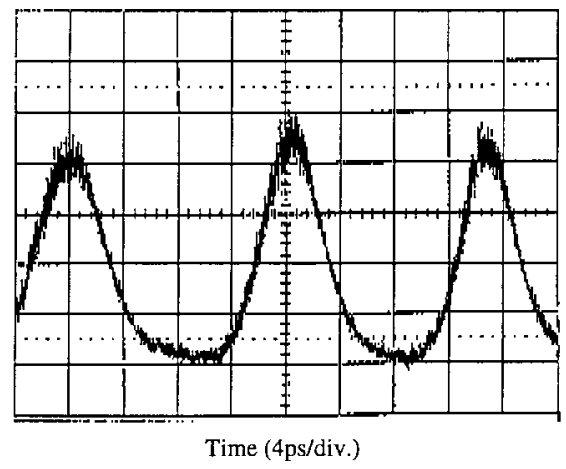

(b)

Fig. 3. Optical pulses with repetition rate of (a) $25 \mathrm{GHz}$ and (b) $70 \mathrm{GHz}$ generated from the same laser device. Horizontal scale: $4 \mathrm{ps} / \mathrm{div}$.

and dispersion, the output from the dual-wavelength laser diode was amplified by an EDFA to $23 \mathrm{dBm}$ and passed through $4.4 \mathrm{~km}$ of dispersion-shifted fiber (DSF), followed by $1 \mathrm{~km}$ of standard single-mode fiber (SMF). In this pulse compression configuration, the dispersion-shifted fiber at the beginning of the system is to generate nonlinear phase modulation. The standard single-mode fiber that followed was to provide a necessary chromatic dispersion to compress the pulse [4]. Fig. 2(a) shows the optical spectrum after the dualwavelength light traveled through the two fiber sections. The initially dual-wavelength light becomes a comb of wavelengths through nonlinear phase modulation process of the nonlinear optical fiber. The corresponding time domain waveform was measured by an autocorrelator based on second-harmonic generation (SHG), and is shown in Fig. 2(b). In this case, the pulse-repetition rate was $50 \mathrm{GHz}$ (20-ps separation between pulses) and the pulse width was approximately $5 \mathrm{ps}$ (FWHM).

As indicated in Fig. 3, adjusting the injection current of the two laser sections allows the pulse-repetition rate to vary continuously. Time-domain waveforms with the pulserepetition rates of 25 and $70 \mathrm{GHz}$ are shown in Fig. 3(a) and (b). Even narrower optical pulses can be expected by selecting precise fiber lengths and using special fibers such as dispersion-decreasing optical fibers [5].

Finally, since the light waves emitted from the two laser sections are mutually independent, their phase noises may cause significant time jitter in the compressed pulse train for practical optical communication applications. This prob- 
lem can be solved by phase locking using a subharmonic modulation on the front section of the laser diode as has been demonstrated in [10]. We are currently working on phase locking and time jitter measurement. The results will be reported elsewhere.

In conclusion, we have demonstrated a novel and simple method to generate tunable-rate, ultrahigh-speed optical pulse trains using a strongly gain-coupled, dual-wavelength DFB laser diode. The repetition rate of the optical pulse train is continuously tunable from 25 to $80 \mathrm{GHz}$ in this particular device, and no high-speed electronics are required in this method. Since the wavelength separation of the dual-wavelength laser diode is determined by the DFB grating design in different laser sections, there is no practical limitation for the maximum wavelength separation and thus high optical pulse repetition rates of up to a terahertz can be easily obtained.

\section{ACKNOWLEDGMENT}

The authors are grateful to M. O'Sullivan of Nortel for many useful discussions.

\section{REFERENCES}

[1] S. Kawanishi, K. Okamoto, M. Ishii, O. Kamatani, and H. Takara, "All-optical time-division-multiplexing of $100 \mathrm{~Gb} / \mathrm{s}$ signal based on four-wave mixing in a traveling-wave semiconductor laser amplifier," Electron. Lett., vol. 11, pp. 976-977, 1997.

[2] D. J. Derickson et al., "Short pulse generation using multisegment modelocked semiconductor lasers," IEEE J. Quantum Electron., vol. 28, pp. 2186-2202, 1992.

[3] A. G. Weber, W. Ronghan, E. H. Bottcher, M. Schell, and D. Bimberg, "Measurement and simulation of of the turn-on delay time jitter in gainswitched semiconductor lasers," IEEE J. Quantum Electron., vol. 28, pp. 441-445, 1992

[4] P. V. Mamyshev, S. V. Chernikov, and E. M. Dianov, "Generation of Fundamental soliton trains for high-bit-rate optical fiber communication lines," IEEE J. Quantum Electron., vol. 27, pp. 2347-2355, 1991.

[5] P. V. Mamyshev, "Dual-wavelength source of high-repetition-rate, transform-limited optical pulses for soliton transmission," Opt. Lett., vol. 19, pp. 2074-2076, 1994.

[6] J. Hong, K. W. Leong, T. Makino, J. Evans, X. Li, and W. P. Huang, "Impact of random facet phase on modal properties of partly gaincoupled DFB lasers," IEEE J. Select. Topics Quantum Electron., vol. 3, pp. 555-568, Apr. 1997.

[7] J. Hong, D. McDonald, F. Shepherd, and T. Makino, "Strongly gaincoupled (SGC) DFB lasers with 10-nm continuous wavelength tuning through single-contact current injection," IEEE Photon. Technol. Lett., submitted for publication.

[8] A. Mecozzi, A, D'Ottavi, and R. Hui, "Four wave mixing in semiconductor lasers operating above threshold," IEEE J. Quantum Electron., vol. 29, pp. 1477-1487, 1993

[9] R. Hui, A. D’Ottavi, A. Mecozzi, and P. Spano, "Injection locking in distributed-feedback semiconductor lasers," IEEE J. Quantum Electron., vol. 27, pp. 1688-1695, 1991.

[10] D. Wake, C. R. Lima, and P. A. Davis, "Optical generation of millimeterwave signals for fiber-radio systems using a dual-mode DFB semiconductor laser," IEEE Trans. Microwave Theory Tech., vol. 43, pp. 2270-2276, Sept. 1995. 